\title{
A single prophylactic dose of ceftriaxone as total antibiotic therapy in transurethral surgery
}

\author{
LEONARD H. FINKELSTEIN, DO, FACOS
}

Ceftriaxone (1 $\mathrm{g}$, intravenous administration, 30 to 60 minutes preoperatively) was the only antimicrobial therapy used for $\mathbf{1 0 3}$ male patients undergoing transurethral surgery. Only patients who had sterile urine were included in the study. Patients with a preoperative catheter placed were excluded. One transurethral incision of the prostate, four direct-vision internal urethrotomies, and 98 transurethral resections of the prostate were performed. Urine specimens for culture were obtained preoperatively and on first voiding after catheter removal for all patients. During the first postoperative visit (11 to 40 days after surgery), urinalysis was performed for all patients, and specimens for culture were obtained from 89 patients. No infections were noted on cultures of first voided specimens after catheter removal; there were four infections $(3.88 \%)$ on first postoperative visit cultures. No significant morbidity occurred secondary to urinary tract infection. According to these findings, the use of antimicrobial agents beyond the preoperative and immediate postoperative period in uninfected patients undergoing transurethral surgery does not appear to be indicated.

The incidence of bacteriuria after prostatectomy has been reported to be $26 \%$ to $70 \%, 1,2$ and, in a significant number of prostatectomy patients with preoperative sterile midstream cultures, bactere- mia has developed postoperatively. ${ }^{3}$ Urethral colonization is considered to be the major cause of urinary tract infection, bacteremia, and fever in the immediate postoperative period. ${ }^{2}$ Contamination by the surgeon and assistants via nose, mouth, and hand to resectoscope as well as the use of teaching attachments whose sterility is not assured also may be contributing factors.

Ceftriaxone, a third-generation cephalosporin, was thought to be applicable as prophylaxis in these cases because of its long half-life and its activity, both in vitro and in vivo, against many strains of Pseudomonas aeruginosa and Serratia marcescens as well as most strains of Enterobacteriaceae, organisms that commonly cause urinary tract infections..$^{4,5}$

Although it has been determined by us ${ }^{6}$ and oth$\mathrm{ers}^{7}$ that a single dose of an antimicrobial agent is effective in preventing postoperative urinary tract infection following transurethral resection of the prostate, many urologists continue to prescribe these agents for as long as four weeks after the surgical procedure. For this reason, a retrospective study was carried out to examine further the efficacy of a single-dose prophylactic antimicrobial agent as total antibiotic therapy in these cases.

\section{Patients and methods}

This retrospective study included all patients operated on in an 18-month-period (July 1986 through December 1987). At our institution, the following protocol is used for all patients undergoing transurethral surgery involving violation of the urothelium: Any patient who is allergic to a cephalosporin antibiotic, who has had an indwelling catheter placed preoperatively, or who has preoperative positive urine culture or bacteriuria is excluded 
from single-dose prophylaxis. One gram of ceftriaxone is administered intravenously 30 to $60 \mathrm{~min}$ utes preoperatively as single-dose prophylaxis.

Urine specimens for culture are obtained from the first or second voiding for all patients after catheter removal. If the test result is positive, appropriate antimicrobial therapy is prescribed. Patients are instructed to return to the office three to four weeks postoperatively, or sooner if necessary. Urinalysis, and, if indicated, urine culture are performed at that time.

Of the 103 patients included in the study, 98 underwent transurethral resection of the prostate, four had direct-vision internal urothrotomy, and one had a transurethral incision of the prostate.

\section{Results}

Two urine cultures obtained from the first voided specimen after catheter removal had $\leqslant 10,000$ colonies. The remainder revealed no growth.

Urinalysis was obtained for all 103 patients on their first postoperative visit (11 to 40 days after surgery). Of the 89 patients who also had urine culture performed during the first postsurgical visit, four $(3.88 \%)$ had positive cultures with $>100,000$ colonies. The organisms isolated were enterococcus ( 2 cases), Enterobacteriaceae (1 case), and Pseudomonas (1 case). All four patients had minimal symptoms and responded to specific therapy guidance after the infectious organism was identified.

There was no prolongation of hospitalization because of urinary tract infections.

\section{Summary}

Based on the findings of this study and on our previous, double-blind, randomized study, ${ }^{6}$ a single dose of ceftriaxone administered intravenously 30 to 60 minutes preoperatively is all that is necessary for antimicrobial protection for patients with sterile urine who are to undergo transurethral surgery.

The use of antimicrobial agents beyond the preoperative and immediate postoperative period does not appear to be indicated. This current widespread practice probably results in a tremendous amount of unnecessary cost.

1. Falkner FR, Ma PT, Murphy DM, et al: Antimicrobial agents for the prevention of urinary tract infection in transurethral surgery. $J$ Urol 1983;129:766-768.

2. Stamey TA: Pathogenesis and treatment of urinary tract infections. Baltimore, Williams \& Wilkins Co, 1980, p 592.

3. Choduk CW, Plant ME: Systemic antibiotics for prophylaxis in urologic surgery: A critical review. J Urol 1979;121:695-699.

4. Angehrn P, Probst PJ, Reiner RI, et al: Ro 13-9904, a long-acting broad-spectrum cephalosporin: In vitro and in vivo studies. Antimicrob Agents Chemother 1980;18:913-921.

5. Neuw HC, Meropol NJ, Fu KP: Antibacterial activity of ceftriaxone (Ro 13-9904), A beta-lactamase-stable cephalosporin. Antimicrob Agents Chemother 1981;19:414-423.

6. Finkelstein LH, Arsht DB, Manfrey SJ, et al: Ceftriaxone in the prevention of postoperative infection in patients undergoing transurethral resection of the prostate. Am J Surg 1984;148:19-21.

7. Ramsey EW, Sheth NK: Antibiotic prophylaxis in patients undergoing prostatectomy. Urology 1983;21:376-378.

From the Division of Urology, Department of Surgery, Osteopathic Medical Center of Philadelphia.

Reprint requests to Dr Finkelstein, GSB Bldg, Suite 714, Belmont and City Avenue, Bala Cynwyd, PA 19004. 